\title{
Dendritic Nanoparticles: The next generation of nanocarriers?
}

Ryan M. Pearson, Suhair Sunoqrot, Hao-jui Hsu, Jin Woo Bae, and Seungpyo Hong*

Department of Biopharmaceutical Sciences, University of Illinois, Chicago, IL 60612

*All correspondence should be addressed to:

Prof. Seungpyo Hong

University of Illinois at Chicago

College of Pharmacy

833 S. Wood St. (MC 865)

Chicago, IL 60612

Phone: $312-413-8294$

Fax: 312-996-0098

Email: sphong@uic.edu 


\section{Summary}

Dendritic polymers have attracted a great deal of scientific interests due to their well-defined unique structure and capability to be multifunctionalized. Here we present a comprehensive overview of various dendrimer-based nanoparticles that are currently being investigated for drug delivery and diagnostics applications. Through a critical review of the old and new dendritic designs, we highlight the advantages and disadvantages of these systems and their structurebiological property relationships. This paper also focuses on the major challenges facing the clinical translation of these nanodevices, and how these challenges are being (or should be) addressed, which will greatly benefit the overall progress of dendrimer-based technologies for theranostics. 


\section{Introduction}

Dendrimers and other dendritic polymers have great potential as multifunctional nano-scale devices because of the uniqueness of their structures and precise controllability over their physical and biological properties. Dendrimers are nanometer-sized (2-10 $\mathrm{nm}$ in diameter), monodispersed, regularly hyperbranched, flexible macromolecules with a large number of peripheral functional groups.[1-3] Unlike conventional hyperbranched polymers, the high level of molecular regularity of dendrimers places these nanomaterials in a unique position to be used for a variety of biomedical applications.[4, 5] The high branching degree of dendrimers enables facile multifunctionalization through conjugation of multiple chemical and/or biological moieties to their surface.[6] Importantly, precisely controlled surface chemistry, along with flexibility and deformability of the dendritic structure, has enabled these molecules to effectively mediate the strong multivalent binding effect that substantially increases binding strength to biological targets.[7, 8] The periphery and two additional functional domains of dendrimers (core and branches) can be tuned to modulate properties, such as: molecular weight, generation (size), surface charge, and functionality as shown in Figure 1A.[1,9] These physical properties play a key role in determining biological properties of the dendrimers such as toxicity, biodistribution, circulation time, intracellular uptake, trafficking and fate.[7, 10, 11] Although further work is still warranted to achieve widespread clinical use, $[1,12]$ dendrimers have shown great potential for clinical translation, as highlighted by several recent reviews.[13-17].

The notable success of dendrimers has inspired the design of various hybrid nanomaterials that incorporate dendritic wedge-shaped sections of dendrimers, or dendrons (Figure 1B). Such designs have been developed with the purpose of combining the advantages of dendrimers with those of other organic and/or inorganic materials.[18, 19] Inclusion of dendrons into polymer 
designs facilitates the synthesis of widely diverse and unique structures that can adopt the multivalent and multifunctional capabilities of dendrimers as summarized in Figure 2. Amphiphilic linear dendron-based block copolymers (LDBC) (Figure 2A-C) are particularly promising because of their ability to be mixed together using a modular approach and to selfassemble into micelles.[18] By forming a core-shell micelle, the hydrophobic core can be used to carry a large number of hydrophobic drug molecules, while a dense hydrophilic layer is projected outward due to the dendritic topology.[20, 21] In addition to micelle formation using LDBCs, unique polymersome-like self-assemblies can be formed from amphiphilic dendrimers (Figure 2G). Developed by the Percec group, Janus dendrimers can self-assemble into nanoscale polymeric vesicles exhibiting low polydispersity and material properties superior to liposomes and conventional polymersomes.[22] These 'dendrimersomes' have received much attention due to their great potential for use as nanocarriers because of their well-controlled structure, high strength, and ability to encapsulate both hydrophilic and hydrophobic drug molecules.[22, 23] Hybrid dendrimer-based nanomaterials can add to the previously enumerated advantages of plain dendrimers and offer unique physicochemical properties that can be functionally tuned to improve upon previously investigated dendritic structures.

The combination of dendrimer-based nanomaterials with various types of polymers is a promising alternative approach that can be used to enhance the therapeutic efficacy and broaden the applications of dendritic materials. For example, our group has been investigating hybrid nanoparticles (NPs) that are a combination of dendrimers and biodegradable polymeric NPs to enhance the control over cellular targeting kinetics of ligand-functionalized dendrimers.[24] Additionally, we have developed a device using antibody-functionalized dendrimers that displayed high sensitivity and selectivity to tumor cells for the early detection of metastatic 
cancer.[8] These alternative uses of dendrimers clearly demonstrate their versatility and their ability to be incorporated into the design of novel functional biomaterials, which has the potential to greatly impact the progression of disease therapy and diagnosis, particularly for cancer.

In this perspective article, we summarize promising dendritic systems that have been designed for drug delivery and diagnostic applications. First, we briefly describe wellestablished synthetic routes of dendrimers. Second, structure-related biological interactions of dendrimers are described to potentiate the importance of how toxicity, biodistribution, cellular uptake, and multivalent binding interactions can be controlled through the appropriate choice of material. We will then continue by discussing the advantages and disadvantages of dendrimers, dendritic-block copolymers, dendrimersomes, and hybrid dendrimer-based nanomaterials with examples to ultimately summarize the challenges regarding the clinical translation of these materials. Lastly, summary and future perspective sections will provide guidelines to follow for developing novel dendrimer-based therapies. 


\section{Synthetic routes of dendrimers}

The controllability of dendrimer synthesis has engendered the development of many diverse dendritic structures with different backbones and surface functional groups. Two major synthetic routes have been developed to synthesize dendrimers as depicted in Figure 3. First developed by Donald Tomalia and his colleagues, divergent synthesis utilizes a polyfunctional core where through successive activation and condensation reactions, the dendrimer grows radially from the core to the surface.[25] In contrast, convergent synthesis, developed by Craig Hawker and Jean Fréchet, enables dendrimers to be synthesized radially from the surface to the focal point.[26] Although the final structures produced using each method are similar, there are pros and cons for each method. Divergent synthesis is most widely adopted and is the preferred method for large scale manufacturing of dendrimers.[2] However, this method often results in dendrimer products with defects such as missing arms and dimers/trimers. This is mostly encountered with higher generation dendrimers, due to the growing number of simultaneous reactions with each generation increase and increasing steric hindrance at the periphery. Using convergent synthesis, dendrimer defects can be minimized because instead of synthesizing the entire dendrimer from one starting point, individual dendrons are produced and purified first before being combined with a polyfunctional core to complete the dendrimer structure.[27] This method, however, still has its disadvantages. For example, the additional number of steps necessary to produce the same dendrimer structure compared to divergent synthesis, the large amount of starting materials required, and low yields when synthesizing larger dendrimers because of steric hindrance at the focal point. For this reason, other synthetic methods have been developed to overcome these shortcomings, such as double-stage convergent growth,[28] orthogonal synthesis,[29] double exponential growth,[30] and orthogonal coupling.[31] 
Regardless of the synthetic route employed, the design of the dendrimer and the resulting parameters such as size, surface charge, and polymer backbone structure are important factors that will ultimately direct its biological interactions. 


\section{Biological Interactions}

Toxicity, biodistribution, and pharmacokinetics

Designing dendritic polymers that exhibit low levels of toxicity with favorable biodistribution profiles is important for these materials to reach clinical translation. Recognizing the importance of achieving controlled biological interactions; this section will provide guidelines as to how dendritic structures can be modulated to achieve this aim. Dendrimer structure-related toxicity and biocompatibility was reviewed thoroughly by Duncan et al.[32] This review provided a summary of additional evidence that supports previous observations by Malik et al.[33] where the most important factors found to impact the cytotoxicity and biocompatibility of dendrimers were surface charge and generation.[32] By far, polyamine dendrimers such as poly(amidoamine) (PAMAM) and poly(propylenimine) (PPI) have been most widely investigated in the literature due to their ease of functionalization through aminebased chemistries and because of their commercial availability. The toxicity of PAMAM dendrimers with increasing generation number can be attributed to the increased number of positive charges per molar concentration and increased contact area of the dendrimer with the cell membrane.[10] It was further shown by Hong et al. using a combination of analytical techniques that the electrostatic interactions of cationic dendrimers with cell membranes induced nano-scale hole formation through reversible (to an extent) membrane destabilization mechanisms.[10, 11, 34] Taken together, these studies by Malik et al. and Hong et al. highlight the need to remove positive charges from the surface of the dendrimer (by acetylation, carboxylation, or PEGylation (coating the surface with poly(ethylene glycol) (PEG))[35] to achieve desirable cytotoxicity profiles. 
To counteract the high toxicity and poor degradability of polyamide dendrimers, alternatively structured dendrimers based on polyester backbones such as 2,2-bis(hydroxymethyl) propionic acid have been prepared.[36, 37] These polyester dendrimers exhibited much lower toxicities due to the reduced non-specific cellular interactions because of the presence of hydroxyl groups on the surface.[38] For example, even at a concentration of $20 \mathrm{mg} / \mathrm{mL}$ after $48 \mathrm{~h}$ incubation with B16F10 melanoma cells, polyester dendrimers maintained approximately $60 \%$ cell viability.[39] The reduction in toxicity is significant compared to amine-terminated dendrimers where $\mathrm{IC}_{50}$ (half maximal inhibitory concentration) values were reported as low as $50 \mu \mathrm{g} / \mathrm{mL}$ after $72 \mathrm{~h}$ incubation using the same cell line.[33] These results emphasize that the use of biodegradable dendrimers lacking positive surface charges (neutral or negative) enhances their biocompatibility. However, the use of surface charge modified-polyamine dendrimers is still appropriate for many biomedical applications and should not be excluded from use based only on this fact.

The biodistribution of dendrimers has been investigated to establish similar relationships with regard to generation and surface composition as accomplished for toxicity. For multimodal imaging, Margerum et al. reported a generation-dependent biodistribution of PAMAM dendrimers that were radiolabeled with gadolinium $(\mathrm{Gd})$ using magnetic resonance imaging (MRI).[40] The liver accumulation of G5 dendrimers after 7 days was significantly higher than lower generations evaluated (G2-4). Due to the need to modify the surface of each dendrimer for imaging purposes, their biodistribution profiles may be significantly altered by the choice of the imaging probe depending on their molecular weight and surface composition. ${ }^{125}$ I-labeled anionic dendrimers displayed 10-20 fold enhancements in circulation times when given to Wistar rats compared to cationic dendrimers.[33] However, both types of dendrimers primarily 
accumulated in the liver after $1 \mathrm{~h}$; thus indicating that the choice of dendrimer surface functionality must be carefully considered to enhance their biodistribution profiles.

PEGylation is another frequently used method to enhance the pharmacokinetic parameters of dendrimers and other nanoparticles by providing stealth-like attributes.[41] Avoidance of opsonization and reduced recognition by the mononuclear phagocyte system (MPS) can ultimately enhance the blood circulation times of dendrimers. For example, PEGylated G4 and G5 Lysine-modified PAMAM dendrimers showed dramatically increased circulation times compared to non-PEGylated dendrimers where only less than $10 \%$ of the injected dose was detected in the blood after 15 min.[42] The authors surmised that the enhancement in circulation time was probably due to the increased molecular weight and high degree of PEG surface coverage. Based on these findings, a full surface coverage of PEG would be important for longcirculating dendrimers and to achieve a favorable pharmacokinetic profile.

As dendron-based materials have been less investigated than dendrimers, generalities with respect to toxicity and in vivo properties cannot be made. Each system is inherently different and therefore it is necessary to evaluate each property on an individual basis to ensure that each desired biological property is satisfied. It is expected, however, that structural properties such as size, backbone composition, and surface charge will follow trends observed with dendrimers. Poon et al. studied the biodistribution of ${ }^{111}$ In-labeled PEGylated G4 LDBC micelles in tumorxenografted nude mice.[43] After 5 days, LDBC micelles accumulated in all vessel-rich organs initially, and most of the micelles were cleared from the body by day 5 . The blood concentration decreased three times more rapidly for LDBC micelles in non-tumored control mice than tumorbearing mice, which was attributed to the accumulation of micelles into the tumor by the enhanced permeability and retention (EPR) effect. Hybrid "bow-tie" dendrimers were 
synthesized by Gillies et al. comprised of two non-symmetric polyester dendrons.[44] The "bow-tie" dendrimers with molecular weights greater than $40 \mathrm{kDa}$ displayed significantly increased circulation times most likely due to the decreased molecular flexibility of higher generation dendrimers and difficulty to be eliminated by the filtration mechanisms present in the kidney as was similarly observed for dendrimers.[42] The conclusions that can be drawn from these studies are that PEGylated dendron-based nanocarriers exhibit low toxicity because of their biocompatible/degradable polyester backbone structure and the lack of high-density positive charges. The presence of high density PEG layers also increases the circulation time by decreasing the clearance rates of the polymers through glomerular filtration mechanisms in the kidney.

\section{Intracellular uptake}

Once a dendritic polymer successfully reaches the appropriate site of action in vivo, it is faced with another barrier, cell entry. For a dendrimer to elicit the desired biological response, whether therapeutic or diagnostic, it is crucial that it be able to cross cell membranes. Many pathways have been proposed as mechanisms responsible for the internalization of dendrimers. It is not surprising that just as toxicity and biodistribution can be controlled through the choice of dendrimer generation, shape, surface charge, and surface ligands, the same can be applicable for internalization mechanisms.[45] Many groups have revealed various mechanisms of PAMAM dendrimer internalization and have been summarized elsewhere.[46] The mechanisms underlying cellular internalization of dendrimers have been the subject of considerable scientific debate. Using Caco-2 cells, Kitchens et al. found that amine-terminated PAMAM and carboxylated PAMAM dendrimers internalized using clathrin-dependent endocytosis.[47] This 
result was independently confirmed using HeLa cells where amine-terminated PAMAM and partially acetylated PAMAM dendrimers internalized using a combination of clathrin-mediated endocytosis and macropinocytosis.[48]

The confusion related to cellular internalization occurred when Perumal et al. showed using A549 cells that carboxylated PAMAM dendrimers internalized via a caveolae-mediated mechanism, while neutral or amine-terminated PAMAM dendrimers internalized using nonclathrin/non-caveolae-mediated mechanisms.[49] Hong et al. studied the cellular internalization mechanisms of amine, carboxyl, and acetylated G7 PAMAM dendrimers.[11] A combination of fluorescently-labeled endocytic markers (cholera-toxin subunit B (CTB), transferrin (Tf), and ganglioside $\mathrm{GM}_{1}$-pyrene) were employed to study the different endocytosis mechanisms of PAMAM dendrimers. As observed using confocal microscopy, amine-terminated G7 PAMAM dendrimers co-localized with $\mathrm{CTB}$ in $\mathrm{KB}$ and Rat2 cells. Acetylated and carboxylated dendrimers did not internalize into these cells at the incubation times tested. CTB internalizes via a caveolae-mediated mechanism after interaction with membrane bound $\mathrm{GM}_{1}$. Thus the colocalization of amine-terminated PAMAM dendrimers with CTB indicated a caveolae-mediated uptake mechanism, which agreed with the results of Perumal et al. To determine the role of $\mathrm{GM}_{1}$, if any, in moderating the internalization of G7 PAMAM dendrimers, experiments were repeated using C6 cells, which are deficient in $\mathrm{GM}_{1}$. Surprisingly, amine-terminated PAMAM dendrimers were still internalized at $37{ }^{\circ} \mathrm{C}$, indicating that there may be multiple internalization pathways activated during dendrimer internalization. Experiments performed at $4{ }^{\circ} \mathrm{C}$ (inhibition of energy dependent cellular uptake) still showed the internalization of amine-terminated PAMAM dendrimers suggesting that the uptake was not necessarily energy dependent. Each of 
the studies mentioned clearly demonstrate that cellular internalization is complex and highly dependent on a number of material properties and cell type.

The conclusion that can be drawn from these studies is that just as the toxicity and biodistribution profiles of different dendritic structures are influenced by material properties, internalization data can be used to design nanocarriers with desired routes of cellular uptake for an additional level of drug delivery, subcellular targeting.[50] In all cases, a new dendrimerbased material should be evaluated individually to ensure an appropriate description of its biological properties.

\section{Multivalent interactions}

Multivalent interactions are characterized by the simultaneous binding of multiple ligands to multiple receptors.[51] These interactions have been found to play a pivotal role in numerous biological processes from adhesion of viral particles to cell surfaces, bacterial, cell-to-cell binding, efficient signaling between cells, antibody-antigen interactions, and prevention of undesired interactions.[51-53] These physiological and pathological processes have spawned the development of many new biomimetic materials attempting to exploit these interactions. Many advantages are observed when using materials that employ multivalent ligands. Most notably, this binding results in an enhancement of binding strength through amplification of pre-existing ligand-receptor interactions.[51, 53, 54] For this reason, ligands for multivalent binding have been engineered into a multitude of nanocarriers with the aim to improve nanocarrier-target cell binding interactions and increase specificity. Dendrimers are an ideal platform to study multivalent interactions because of their high number of surface groups and ability for structural deformation to optimize ligand orientation and maximize substrate binding.[55] The earliest 
studies describing the multivalent binding of dendrimers were performed using mannosidefunctionalized dendrimers. When 16 mannoside moieties were conjugated to the dendrimer, a significant enhancement in the binding avidity towards concanavalin A (Con A) and pea lectins was observed compared to single binding pairs.[56] However, the enhancement in binding avidity can only be increased to a certain level before saturation of available binding sites and/or steric hindrance from additional ligands on the dendrimer surface will negatively affect binding.[57]

To enhance the multivalent binding effect, incorporation of flexible chemical linkers such as PEG can confer preferred geometric orientation of ligands towards their appropriate receptor in a thermodynamically favorable manner.[58-61]. Ligand numbers and parameters of the polymer such as size, shape, and density of ligands can greatly affect multivalent interactions. To investigate the role of polymer architecture and ligand presentation on receptor binding, Gestwicki et al. evaluated a set of 28 mannose-containing ligands, which varied in size, shape, and number.[62] Ligands from five architectural classes (low molecular weight, dendrimer, bovine serum albumin (BSA), linear, and polydisperse polymers) were subjected to highthroughput assays to evaluate receptor clustering (number and rate) and receptor proximity to Con A. The results obtained indicate that the number of ligands and their surface presentation were the most important parameters, in addition to the multivalent ligand architecture.

Dendrimer-mediated multivalent interactions can also have a significant impact on improving the efficacy of targeted drug delivery systems for the treatment of cancer.[7, 8, 63-66]. To quantify the dendrimer-mediated multivalent binding effect, Hong et al. measured the binding avidities of G5 PAMAM dendrimers functionalized with different numbers of folic acid (FA) using surface plasmon resonance (SPR).[7] Binding avidities of multi-functionalized dendrimers 
towards folate binding protein (FBP) were enhanced up to 170,000-fold greater compared to free FA. However, increased numbers of FA conjugated per dendrimer $(\sim 7 \mathrm{FA})$ led to the deterioration of material properties (increased polydispersity index (PDI) and poor water solubility). Using fluorescence-activated cell sorting (FACS), saturation behaviors were observed in KB cells overexpressing FA receptor (FAR). When the number of FA per dendrimer was increased to over 7, marginal improvement in cell binding was observed. These results indicate that there is an optimal number of ligands that should be conjugated per dendrimer to achieve maximal multivalent binding effect without decreasing the structural homogeneity of the materials. Therefore, to optimize multivalent interactions for any material, architectural parameters of the mediator such as size, shape, and surface ligand density should be considered critically.[62] Additionally, the incorporation of flexible chemical linkers and less than complete surface coverage by ligands would likely increase the multivalent binding effect. Overall, the large variation in materials, ligands, and receptors indicates that multivalent interactions should be optimized on a case-by-case basis. 


\section{Advancement of dendritic designs for drug delivery and other applications}

\section{Dendrimers}

\section{Advantages and Disadvantages}

The success of dendrimers as targeted drug delivery vehicles has fostered their use in a variety of therapeutic applications from cancer therapy to diagnostic imaging.[13, 15, 46, 67, 68] For biological applications in particular, dendrimers possess a number of advantages in terms of both structure and function. The well-defined architectures, precise controllability of the functionality of their large number of surface groups, and high deformability allow their biological interactions to be engineered at a molecular level. The synthesis of dendrimers with extremely low PDIs (1.0005 to 1.1)[2] can now be easily achieved at large scales, which separates them from other nanocarriers based on conventional polymers (note that most of the condensation polymers typically exhibit PDI values higher than 2.0). The well-defined architectures offer great promise because of their ability to be characterized with minimal batchto-batch variations, which could ultimately facilitate their translation to the clinic. Due to the vast array of small chemical building blocks that dendrimers can be synthesized from, functions such as biodegradability, stimuli-responsiveness $(\mathrm{pH}$, temperature, enzyme, reducible, ultraviolet light), and surface functionality (targeting, imaging, therapeutic) can be incorporated into their

designs. Notably, the flexibility of their structure has been shown to enable the formation of strong multivalent interactions between surface-conjugated ligands and receptors, leading to enhanced targeting efficacy of drug payloads.[7, 69]

Despite the advances made with dendrimers as drug delivery vehicles, they are still faced with challenges related to synthesis and in vivo performance. For example, the multiple steps involved in the synthesis of multifunctional dendritic nanodevices can often result in great batch- 
to-batch variability and deterioration of the material's homogeneity.[70] The Baker group developed a one-pot synthetic approach to address this issue.[71] By precisely controlling the mixing ratio of ligand to dendrimer, well-defined materials can be created without the need for sequential conjugation and purification. Another potential pitfall of dendrimer conjugates is that the attachment of multiple molecules can often result in a population of conjugates with a wide distribution of number of ligands where the arithmetic mean of the number of ligands does not necessarily represent the most common species in the population.[72] This could potentially lead to inconsistent and sometimes biologically inactive batches of conjugates during large-scale production. In a recent attempt to achieve better control over dendrimer-ligand distribution, FAtargeted dendrimer-MTX conjugates were synthesized with precisely controlled ratios of FA and MTX, by attaching those molecules to a triazine linker first, followed by conjugation to dendrimer surfaces by click chemistry.[73]

The in vivo fate of targeted dendrimer conjugates remains a challenge by itself. Due to their relatively small size, dendrimers are easily excreted through the renal route.[69, 74] Attachment of targeting ligands such as FA has also resulted in significant liver accumulation.[69] Surface modification by PEGylation is one approach by which the blood circulation time of dendrimers can be prolonged, which is thought to occur through a combination of increased molecular weight and size of the dendrimers.[42, 75]

\section{Applications}

Table 1 summarizes the recent advances in the in vivo applications of different types of dendrimers. PAMAM dendrimers are among the most widely investigated family of dendrimers for drug delivery. The internal tertiary amines and amide linkages also contribute to their unique architecture, allowing their cavities to host metals or other guest molecules. One of the early 
attempts to use PAMAM dendrimers as targeted MRI agents was using Gd-complexed, FAconjugated dendrimers to target tumor tissues with high expression levels of FAR.[76] The first reported in vivo antitumor efficacy of PAMAM dendrimers as anticancer drug carriers was using cisplatin-conjugated G4 dendrimers.[77] The conjugates (30-40 $\mathrm{nm}$ in size) passively targeted to murine subcutaneous tumors through the EPR effect. This allowed tumor levels of platinum to be fivefold higher than the free drug, and led to a significant reduction in tumor growth rate compared to unconjugated cisplatin. FA-targeted, fluorescein isothiocyanate (FITC)-labeled PAMAM dendrimers have also been investigated in vitro and in vivo for the same purpose, while also delivering therapeutic agents such as methotrexate (MTX) and Taxol.[69, 78, 79] In a mouse xenograft model of human KB tumors overexpressing FAR, FA-targeted MTXconjugated dendrimers selectively accumulated and were taken up by KB cells, leading to a 510-fold increase in intracellular dendrimer concentration compared to non-targeted dendrimers.[69] However, the small diameter of the dendrimer conjugates $(<5 \mathrm{~nm})$ resulted in rapid renal clearance, which demonstrates the need for structural modification to prolong the in vivo circulation times in order to maximize their efficacy.

Positively charged dendrimers have also been investigated for gene delivery applications.[80] These efforts have resulted in the production of commercially available dendrimer-based transfection kits (SuperFect ${ }^{\circledR}$, Qiagen, Hilden, Germany). Successful inhibition of tumor growth and angiogenesis has also been reported using dendrimers as gene delivery vectors in a murine xenograft model.[81] However, the major hurdle facing the successful in vivo application of these nanocarriers for gene delivery remains the toxicity associated with the strong cationic surface charge of the dendrimers, $[10,11,34]$ which is essential for their ability to condense the genetic material. Modification of the structure may be able to solve these issues, but in order for 
this technology to reach clinical relevance, careful optimization of the polymer properties and transfection efficiency needs to done order to harness the full potential of dendrimer-based nonviral gene delivery vectors. 
Dendritic-block copolymers

Advantages and disadvantages

As highlighted in previous sections, dendrimers provide many advantages over current therapeutic and diagnostic devices. Synthesis of hybrid nanomaterials with similarly controlled molecular properties as dendrimers can be achieved through the combination of dendrons with other polymeric materials. One of the most interesting types of dendronized materials is the LDBC.[18] These materials can combine the advantages of dendrimers (mentioned in the previous section) with those of linear block copolymers (high drug loading and modularity). The appropriate engineering of hydrophilic-lipophilic balances (HLB) into each LDBC will allow self-assembled structure formation to occur (Figure 4A).[20, 21, 82] Unlike dendrimers, which are small $(<10 \mathrm{~nm})$ and unimolecular, dendron-based micelles are larger (typically $>20 \mathrm{~nm}$ ) due to their multimolecular nature, and can encapsulate hydrophobic drugs within their core during the self-assembly process. Their increased size allows passive targeting of the nanocarriers to tumor sites, which possess leaky vasculature and decreased lymphatic drainage by the EPR effect and encapsulation of drug molecules within the nanocarrier has certain advantages compared to covalent conjugation. Drug encapsulation does not involve any chemical modification of the drug molecules and eliminates the need to present potentially antigenic molecules at their surface that can result in increased in vivo clearance.[69] This drastically simplifies the manufacturing process and decreases the potential of observing reduced efficacy or toxicity of the delivered drugs because of the alteration of the chemical structure of the drug. However, it should be noted that the release kinetics of encapsulated drug molecules are often more difficult to control than drug-conjugates;[83] but the HLB of the nanocarrier can be used to control the drug release.[20] To enhance the delivery of the encapsulated drug payloads, targeting ligands can be 
added to dendritic-block copolymers. The modularity of their design can allow similar studies to those of Hong et al.[7] to be performed to find the optimal density of targeting ligands on the surface of dendron-based micelles.[21,43] This indicates that the micelles formed by dendriticblock copolymers still preserve the multivalent targeting effect of dendrimers. A previous report by our group has identified key advantages of PEGylated dendron-based copolymers (PDC) compared to linear-block copolymers at similar HLBs.[20] The preorganized conical molecular architecture of each PDC provided a decreased entropic cost for self-assembly resulting in 1-2 orders of magnitude lower CMCs observed for PDC micelles compared to linear copolymer micelles at similar HLBs (Figure 4B). Molecular dynamic simulations of the micelle structures showed that the surface of the PDC micelles was completely covered by a PEG layer, whereas a significant portion of the hydrophobic core of the linear copolymer micelle was observed at the surface. This high surface coverage by a short non-fouling PEG layer is highly beneficial to enhance the in vivo circulation time of nanoparticles.[84]

It should also be noted that nanocarriers formed by micellization of dendritic-block copolymer still have certain innate disadvantages that are similar to polymeric micelles. First, the combination of dendrons with linear polymers will certainly increase the PDI of the block copolymer, which could affect the reproducibility of the nanostructures formed. Secondly, although dendritic-block copolymer micelles can provide lower $\mathrm{CMCs}$ than linear-block copolymer micelles, their structural stability in the blood has only been investigated in a few studies.[43, 85] Lastly, these nanocarriers can only encapsulate relatively hydrophobic drugs due to the hydrophobic nature of the core, making the loading of hydrophilic drugs a challenge, unless they are conjugated to the surface of the nanocarrier. 


\section{Applications}

For drug delivery applications, hydrophobic polymers can be dendronized at one end and conjugated with a hydrophilic polymer on the branches to form an amphiphilic copolymer. As we reported, the conical structure of the dendron stabilizes the structure of the micelles and results in extremely stable structures $\left(\mathrm{CMC} \sim 10^{-8} \mathrm{M}\right)$, [20] which is important for micellar drug delivery carriers due to the immediate dilution factor upon injection into the blood stream. Furthermore, the enhancement in the targeting effect provided by the optimization of ligand clusters on the micelle surface demonstrates the great potential of these novel nanocarriers to be more effective than linear-block copolymer micelles for targeted drug delivery.[21, 43]

Alternatively, the dendron can be used to mediate the conjugation of multiple hydrophobic moieties to a single hydrophilic tail (Figure $2 \mathrm{C}$ and F). $[82,86]$ Using this approach, linear PEGblock dendritic cholic acid (CA) copolymers, or telodendrimers, for the delivery of paclitaxel targeting the overexpression of $\alpha-3$ integrin on ovarian cancer cells.[85] These targeted telodendrimers exhibited superior antitumor efficacy in mice bearing SKOV-3 xenografts compared to non-targeted telodendrimers and the clinical formulation Taxol. Dendritic block copolymers have also been used for gene delivery purposes.[87-91] In this case, positively charged branches are needed for the dendritic copolymers to form a complex with negatively charged DNA. By conjugating the dendron with a hydrophilic polymer such as PEG, it forms a PEG layer on the complex surface which further protects the DNA from enzymatic cleavage and prolongs the circulation time in vivo by shielding the positive charge. Targeted gene delivery can also be achieved by incorporating targeting ligands or tumor homing peptides into the design of the dendron-based gene delivery platforms.[91, 92] Dendron-based drug delivery systems 
represent a novel class of materials that can be used to potentiate the advancement of targeted therapies from the bench to the clinic. 
Dendrimersomes

Dendrimersomes are self-assembled, double-layered vesicles formed from amphiphilic Janus dendrimers.[22] Percec et al. prepared a total of 107 amphiphilic Janus dendrimers from 11 distinct libraries. Unlike liposomes or polymersomes, dendrimersomes can be easily prepared using the ethanol injection method and form stable and monodisperse vesicles that have mechanical stability and impermeability to be applied as a drug carrier. Through their unique well-organized structure, dendrimersomes combine the structure and function of liposomes, with the mechanical properties and stability of polymersomes. In addition, by selecting the type of Janus dendrimer, $\mathrm{pH}$-dependent drug release can be achieved to target subcellular compartments. Fluorescent dye-conjugated and blank Janus dendrimers can be combined in a modular fashion to form unilamellar fluorescent-labeled dendrimersomes without affecting their morphology, indicating that they can also be used for diagnostic imaging purposes. The size and material properties of many of the dendrimersomes synthesized was evaluated using a variety of experimental techniques.[23] With a fixed hydrophobic alkyl chain length, the most stable and largest dendrimersomes were the ones with the thinnest membrane, which is attributed to the large degree of interdigitation between the alkyl groups of the Janus dendrimers. Although our understanding of dendrimersomes is still limited, these nanocarriers present are a versatile platform in addition to the amphiphilic LDBCs that can be further engineered for biomedical applications. 
Other dendrimer-based nanomaterials for improving cancer diagnosis and therapy

Dendrimer-based nanodevices for circulating tumor cell (CTC) capturing

The presence of circulating tumor cells (CTCs) in the peripheral blood of cancer patients has been found to be of prognostic significance for different types of solid tumors such as breast cancer.[93, 94] The detection and characterization of these cells is a promising method for both diagnosis and clinical management of cancer patients as well as monitoring treatment. The frequency of CTCs in peripheral blood can be as low as one out of a billion $\left(10^{9}\right)$ hematologic cells. This represents a major challenge for the available analytical methods, and necessitates the development of a very sensitive and highly specific detection strategy in order to achieve clinically significant CTC detection.

Previous efforts to increase the sensitivity of CTC devices were engineering-based, such as topographical modification of surfaces[95] and chaotic mixer fluidics.[96] In a recent report by our group,[8] a novel approach for the enhanced capture of CTCs has been investigated using a dendrimer-based biomimetic device that takes advantage of naturally occurring processes such as multivalent binding and cell rolling (Figure 5).[97, 98] Using generation 7 (G7) PAMAM dendrimers coated with anti-epithelial cell adhesion molecule (aEpCAM) (a commonly used antibody for CTC capturing),[95, 96, 99] our CTC capturing device also combined the physiologic process of cell rolling mediated by E-selectin. The latter approach has been applied to further enhance the sensitivity and specificity of the device towards tumor cells, by mimicking the process of CTC recruitment to the endothelium. $[8,100]$

Using SPR, the binding avidities between G7-aEpCAM conjugates and EpCAMimmobilized sensor chips were significantly increased compared to free aEpCAM by $10^{6}$-fold, which translated into enhanced binding stability of tumor cells on the dendrimer-functionalized 
surfaces during flow chamber experiments. Additionally, the biomimetic combination of multivalent binding and E-selectin-mediated cell rolling resulted in substantially enhanced tumor cell detection. This example demonstrates the great potential for dendrimers in bringing together nanotechnology and biomimicry for the advancement of existing diagnostic and prognostic tools. 
Hybrid nanoparticles using targeted dendrimers and polymeric nanoparticles

Polymeric nanoparticles (NPs) have also demonstrated great potential for targeted drug delivery to tumors. As with dendrimers, the clinical application of these nanocarriers has been hindered by the lack of control over their targeting kinetics. Surface decoration of polymeric NPs and other nanocarriers such as liposomes with targeting agents can also lead to MPS uptake and rapid clearance, despite the presence of PEG outer layers.[101, 102]

In order to exploit the strengths and to address the limitations of the two nanocarriers (dendrimers and polymeric NPs), our group has designed a novel multi-scale hybrid NP (nanohybrid) platform that allows targeting kinetics to be effectively controlled.[24] The nanohybrid platform consisted of FA-targeted generation 4 (G4) PAMAM dendrimers encapsulated within poly(ethylene glycol)- $b$-poly( $D, L$-lactide) (PEG-PLA) NPs (Figure 6). Through this design, the encapsulated targeted dendrimer conjugates selectively interacted with FAR-overexpressing $\mathrm{KB}$ cells upon their release depending on the MW of the encapsulating copolymers, which determined their targeting kinetics. The nanohybrid system provides evidence that selective cellular interactions of actively targeted polymer conjugates can be kinetically controlled to potentially enhance dendrimer targeting to tumors in vivo. We expect that the biodegradable shell (e.g. PEG-PLA), can protect the targeted dendrimers from nonselective interactions and also premature elimination, as what has been previously observed with targeted nanocarriers with surface-exposed ligands.[103] The multiscale design afforded by the nanohybrid system can also enable the sequential utilization of passive and active targeting. By controlling the size of the hybrid NPs, passive accumulation at the tumor site is made possible through the EPR effect. This is followed by the controlled release of targeted 
dendrimers, which allows active targeting to individual tumor cells, with the advantage of multivalent binding and more efficient tumor penetration due to their small size.[104]

\section{Challenges with clinical translation of dendrimer-based nanodevices}

Dendrimer-based technologies for cancer therapy and diagnosis have made significant progress over the past two decades. However, the advancement of clinical trials is still hindered by several limitations. Some of these issues include the sometimes complex, multistep procedures involved in the synthesis and processing of dendrimer-based nanoparticles. The covalent attachment of drug payloads, which presents a more controllable approach to drug loading and release compared to simple dendrimer-drug inclusion complexes, can result in limited drug loading capacities and increased amounts of polymers to be injected to reach a therapeutic dose of drug. Excessive conjugation of drug and other molecules to the surface of dendrimers can also induce undesirable changes in the material properties and polydispersity.[1, 7, 105] Additionally, issues pertaining to the biodistribution and the in vivo fate of dendritic nanomaterials $[75,106]$ need to be properly addressed, which will influence the successful translation of these therapies from the bench to the clinic. Lastly, the major obstacle that these technologies need to overcome in order to reach widespread clinical use is the poor and sometimes unpredictable correlation between in vivo efficacy in animal models and in humans. Even if the animal studies demonstrate enhancement in therapeutic efficacy, this enhancement needs to be significant enough so that it is still maintained when translated into humans. 


\section{Summary}

The great potential of dendritic nanomaterials has been demonstrated through numerous examples. Implementation of rational design strategies as outlined in the previous sections will result in the progression of these materials to clinical use. Biological properties of dendrimers such as: toxicity, biodistribution, and intracellular uptake have all been successfully controlled by the appropriate choice of dendrimer generation (size), backbone, and surface composition. Optimization of multivalent interactions can substantially enhance active targeting of dendritic nanoparticles by exponentially increased targeting ligand-receptor binding avidities (up to 170,000-fold).[7] This can be exploited to ensure the dendrimer persistence in the tumor site and increase cellular uptake. The design of bio-applicable dendrimers should also include a neutral surface charge, an optimized density of targeting ligands, a hydrophilic PEG layer (to increase size for passive targeting and increase blood circulation times), and a combination of therapeutic (chemical- and/or nucleic acid-based) and diagnostic (fluorophore/MRI) molecules to endow multifunctionality to the dendrimer.

Alternative dendrimer-based nanomaterial such as LDBCs-based micelles, dendrimer-coated surfaces, and hybrid NPs are the examples of recent development in the field of nanomedicine. The ease with which different LDBCs can be combined to form multifunctional micelles is advantageous to develop personalized therapeutics for the treatment of a variety of diseases. Dendrimer-based CTC detection devices have great promise for the diagnosis of early metastatic disease. The results obtained from such a sensitive and specific device will ultimately decrease the time necessary to diagnose tumor metastasis by clinicians, subsequently enhancing patient treatment and probably life span. Hybrid NPs offer some unique design characteristics such as controllable targeting kinetics by first accumulating into tumor sites by passive targeting (using 
polymeric NPs), followed by release of actively targeted dendrimer-based therapeutics. Each of the nanotechnologies described here will have a significant impact on how targeted therapies and detection devices are designed and translated in the future. 


\section{4) Future perspective}

Dendritic nanomaterials are making a high impact on the field of nanomedicine, and will continue to do so for years to come. As demonstrated in this perspective article, dendritic nanomaterials offer many advantages for effective cancer diagnosis and therapy. Although their

clinical translation has been slower than expected due to issues in toxicity, scalability, and reproducibility, the ever-growing development in dendrimer chemistry, together with extensive biological validation, will produce more biocompatible, scalable, and reliable dendrimer-based devices. This in turn will help address the challenges on the road to their successful clinical applications, resulting in profound impact on human health. 
Table 1. Recent advances in in vivo biological applications of dendrimers.

\begin{tabular}{lll} 
Application & Dendrimer chemistry & References \\
\hline Anticancer drug & PAMAM & {$[69,75,77,107,108]$} \\
delivery & & \\
& PE & {$[39]$} \\
& Polypeptide & {$[109,110]$} \\
& PLL & {$[111]$} \\
& PEA & {$[112]$} \\
& PEPE & {$[113]$} \\
\hline Gene delivery & PAMAM & {$[81]$} \\
& PPI & {$[114]$} \\
\hline Drug/Vaccine & PLL & {$[115-119]$} \\
\hline Bioimaging & PAMAM & {$[40,76,120-122]$} \\
& PLL & {$[123,124]$} \\
& PPI & {$[125]$} \\
& PE & {$[126,127]$} \\
\end{tabular}

Poly(amidoamine); PAMAM, Poly(l-lysine); PLL, Poly(propylenimine); PPI, Polyester; PE, Polyether-copolyester; PEPE, Poly(ester-amide); PEA. 
A

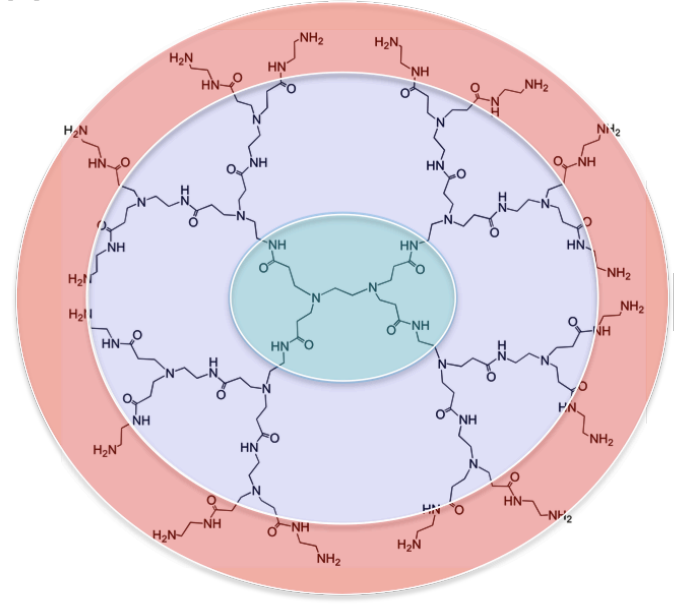

B

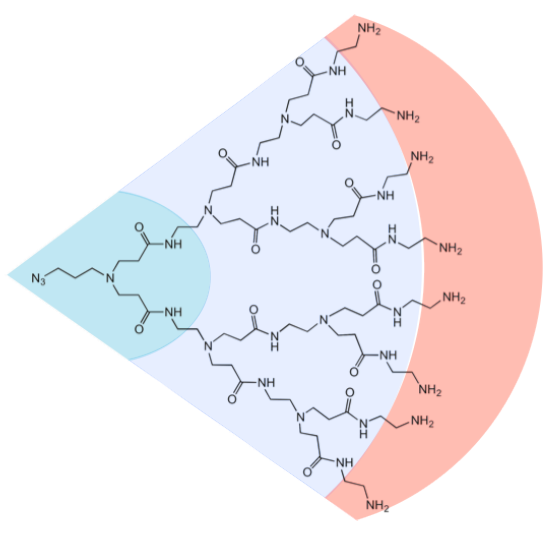

Figure 1: Chemical structures of the PAMAM (A) dendrimer and (B) dendron. The shaded regions represent the three domains of each structure (blue; core, purple; branches, red; periphery). 
A.

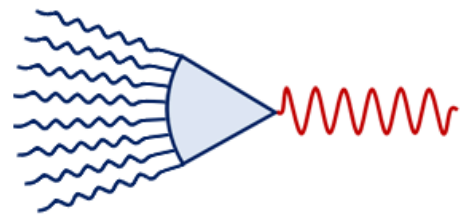

B.

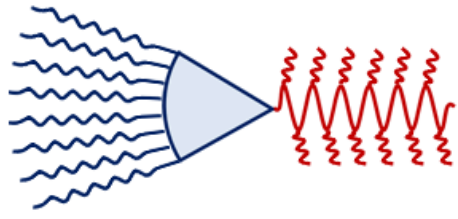

C.

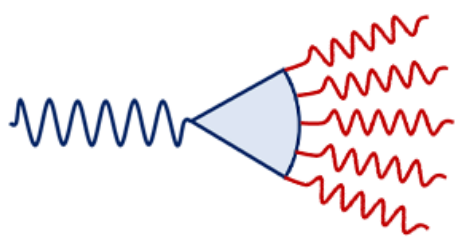

D.

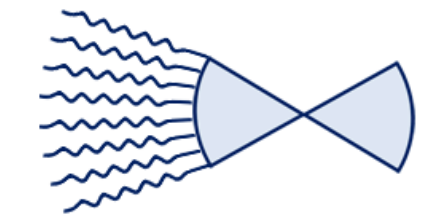

E.

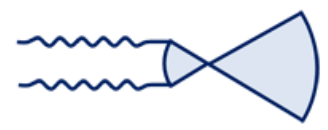

$\mathrm{F}$.

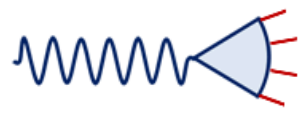

G.

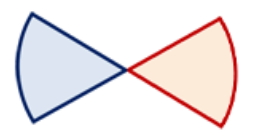

$\mathrm{H}$.

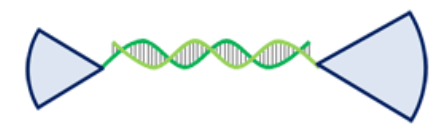

I.

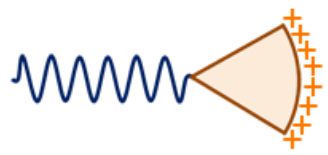

J.

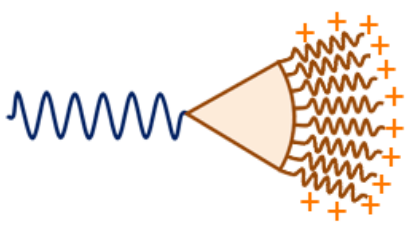

Figure 2: Various dendritic structures: (A) Dendritic block copolymer with multiple hydrophilic chains and single hydrophobic chain,[20] (B) Tree-shaped comb-dendritic block copolymer,[128] (C) Dendritic block copolymer with multiple hydrophobic chains and single hydrophilic chain,[86] Bow-tie shaped dendritic scaffold I (D) and II (E),[129] (F) telodendrimer,[82] (G) Janus dendrimer,[22] (H) ssDNA functionalized dendron,[130] (I) PEGylated PAMAM dendron,[90] and (J) Modified PAMAM dendron with hydrophilic tail.[91] 


\section{Divergent Synthesis}

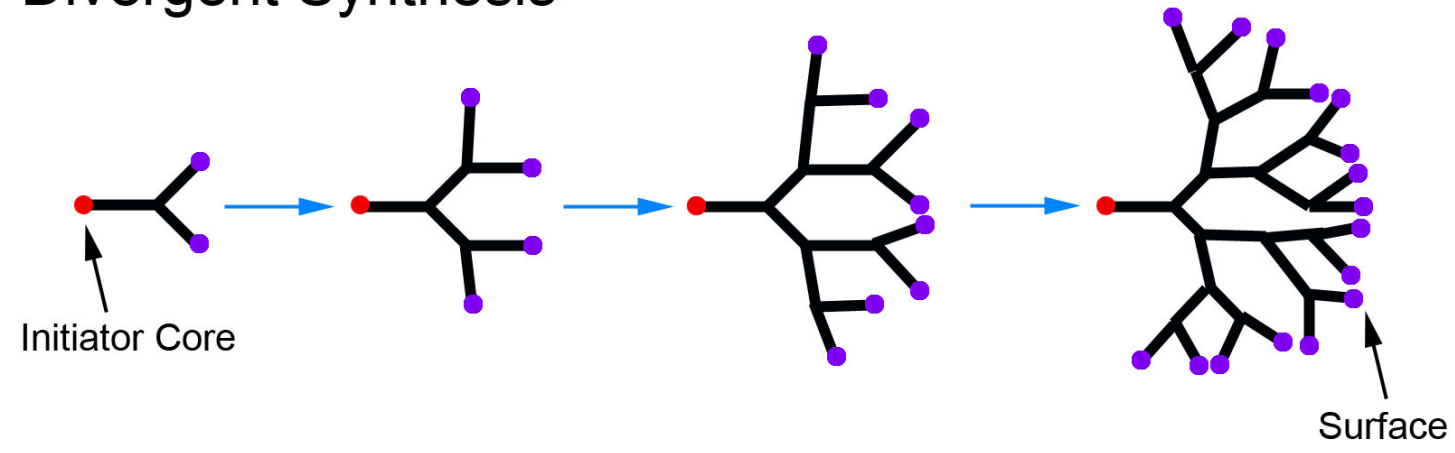

\section{Convergent Synthesis}

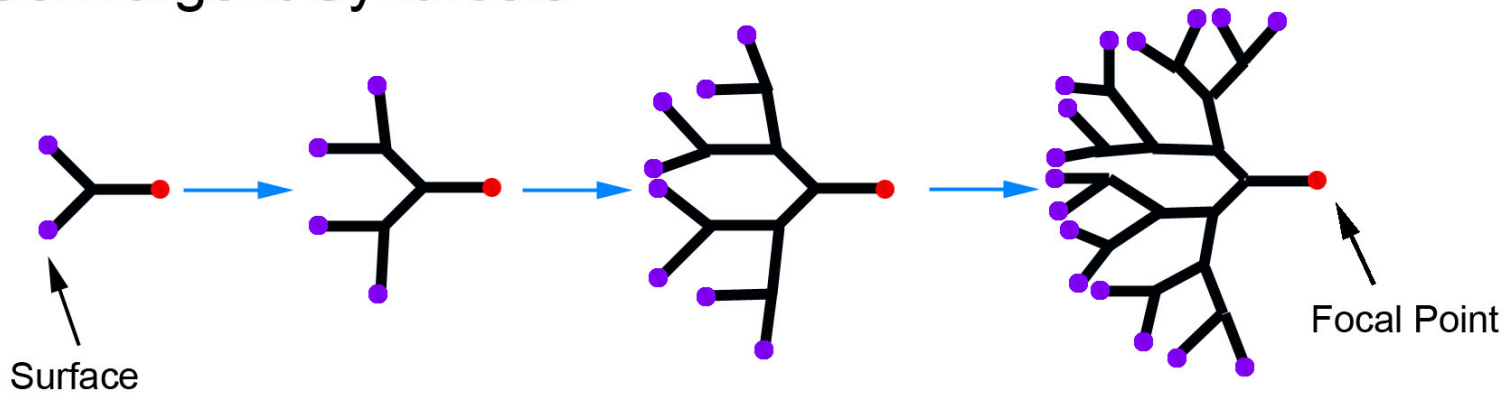

Figure 3: Major synthesis routes for synthesis of dendrimers and other dendritic polymers. In divergent synthesis, the dendrimer grows radially from the core to the surface. In convergent synthesis, individual dendrons are synthesized from the surface to the focal point and are combined in the final step. This figure is adapted from ref.[2] with modification. 


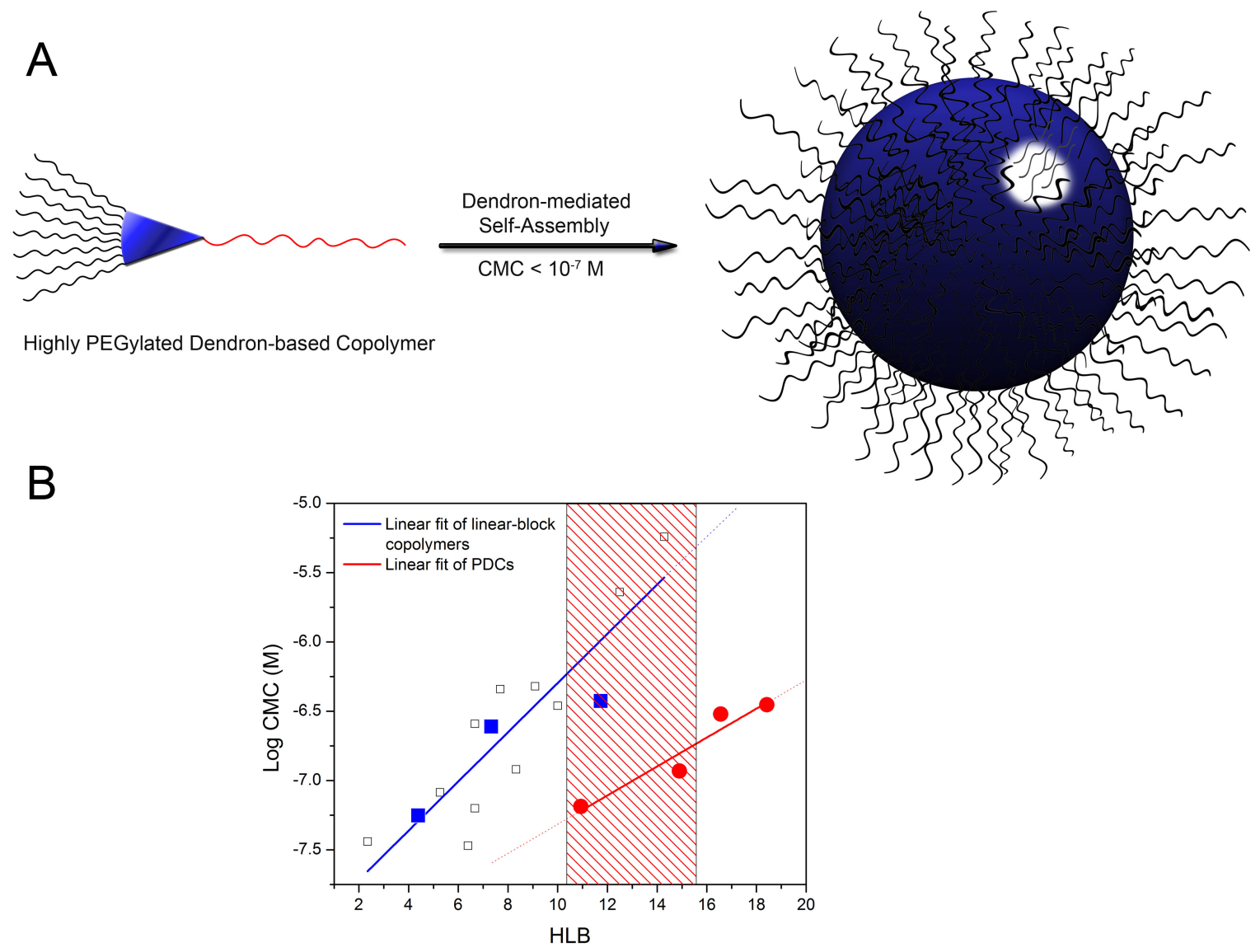

Figure 4. Self-assembly of dendron-based copolymers. (A) The conical molecular architecture of the PEGylated dendron-based copolymer (PDC) facilitates the formation of highly PEGylated micelles, (B) critical micelle concentration (CMC) versus HLB for linear-block copolymers and PDCs demonstrating the remarkable thermodynamic stability of PDCs at high HLBs [Bae et al. (2011). Chemical Communications, 47, 10302-10304] - Reproduced by permission of The Royal Society of Chemistry.[20] 
A
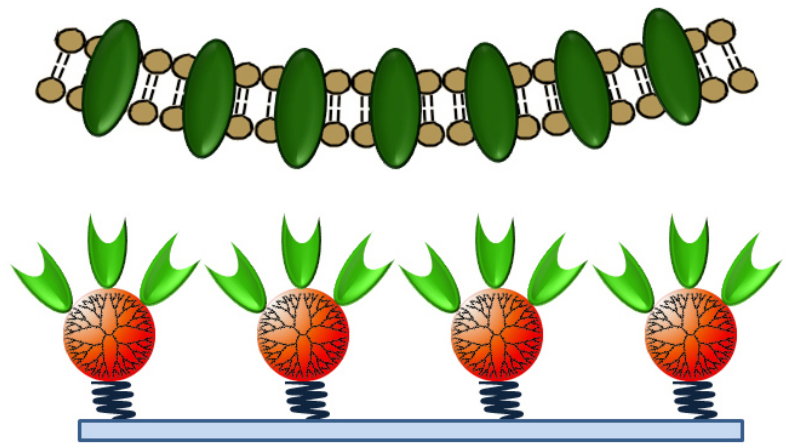

B
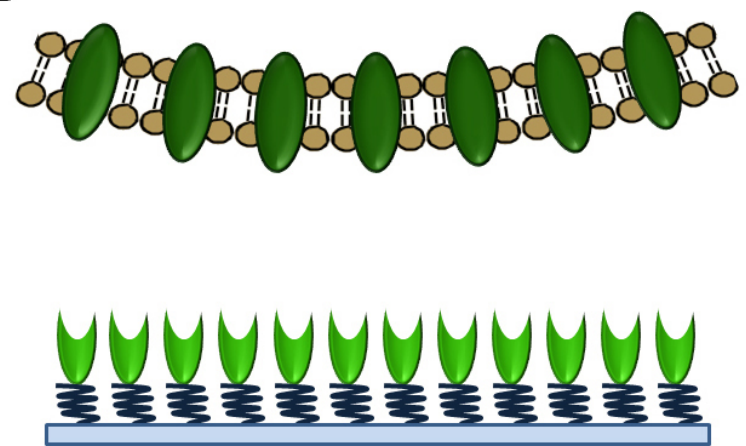

Figure 5: Dendrimer-mediated multivalent capture of cancer cells. (A) aEpCAM-conjugated G7 PAMAM dendrimers immobilized on a PEGylated glass surface, (B) aEpCAM directly immobilized on the PEGylated glass surface. This figure is adapted from Myung et al.[8] with modification. 


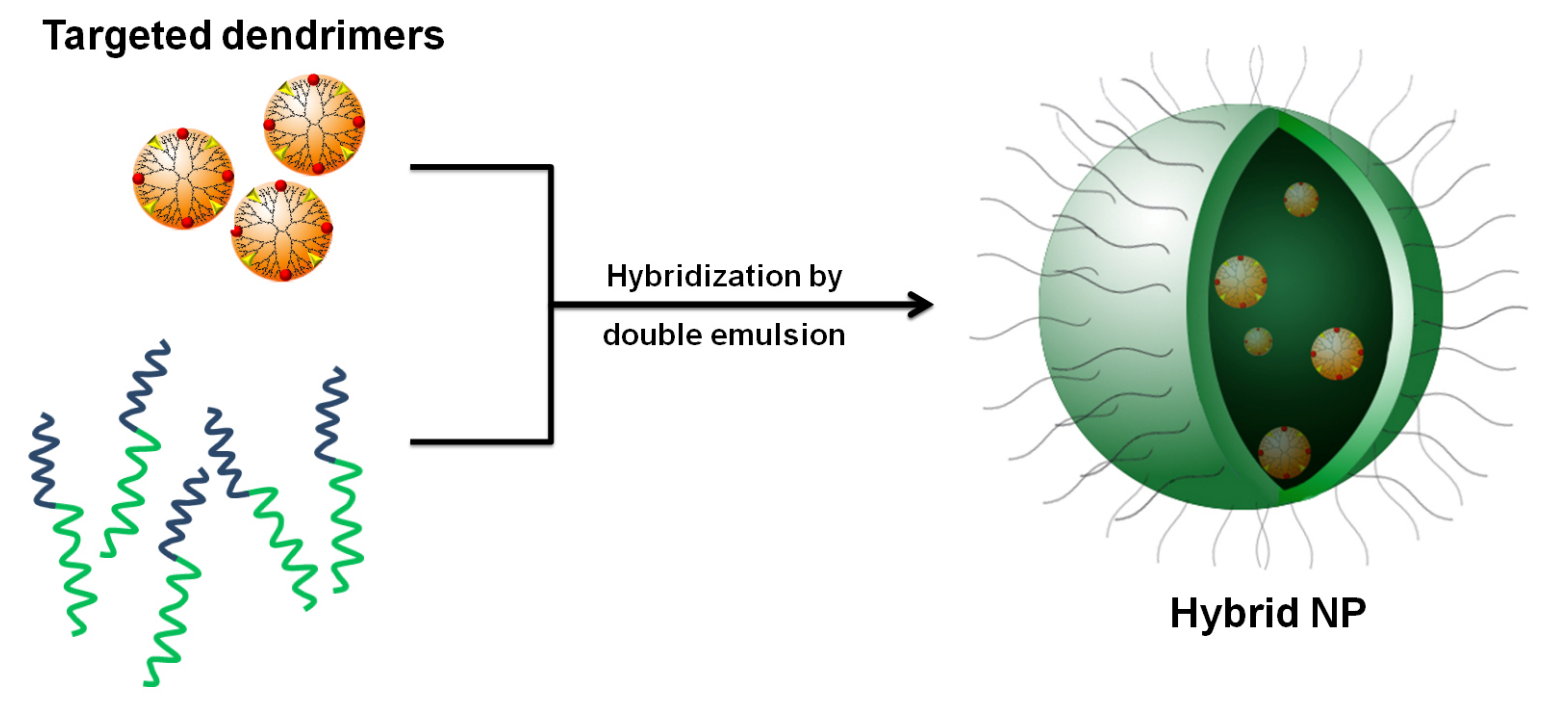

\section{PEG-PLA copolymers}

Figure 6: Hybrid NPs of FA-targeted dendrimers and PEG-PLA copolymers for temporally controlled targeting to FAR-overexpressing cancer cells. Reprinted (adapted) with permission from (Sunoqrot et al. (2012). Biomacromolecules, 13, 1223-1230). Copyright 2012 American Chemical Society. 
Per the guidelines for Therapeutic Delivery, below are the Key Terms with definitions/facts, Executive Summary, and Reference Annotations

Key Terms (5-10 key words/technical terms/definitions)

Dendrimer - A word derived from Greek origin 'dendros' meaning trees, and 'meros' meaning parts. It speaks to its highly branched, symmetrical architecture.

Hyperbranched polymer- Polymers synthesized from condensation or addition polymerizations using monomers with more than 2 functional groups, yielding highly branched, irregular structures.

Biodistribution - The accumulation and distribution of compounds of interest within organs of living animals after administration.

Hybrid nanomaterials - A combination of multiple organic and/or inorganic components into a single nano-scale material.

Dendron - A single branch of a dendrimer, which is usually synthesized using the Convergent synthesis method.

Core-shell micelle - A self-assembled structure produced by amphiphilic copolymers or lipids where a water-insoluble component forms the core and a water-soluble component forms the shell.

Nanocarrier - A nano-sized drug delivery system capable of performing a targeted therapeutic and/or diagnostic function with a size typically less than $200 \mathrm{~nm}$.

Hybrid nanoparticle - The result of the combination of multiple polymeric components into a multilayered, structurally well-defined nanocarrier.

Biocompatibility - A generic term describing biological properties for a material in terms of toxicity and immune responses to biological systems

Binding Avidity - The combined strength of multiple simultaneous ligand-receptor binding interactions - not to be confused with affinity, which refers to a single binding interaction.

Polydispersity Index (PDI) - A measure of the molecular weight distribution of polymeric materials defined as weight-average molecular weight divided by number-average molecular weight $\left(\mathrm{PDI}=\mathrm{M}_{\mathrm{w}} / \mathrm{M}_{\mathrm{n}}\right)$. PDI of 1.0 indicates monodispersity. 


\section{Executive Summary}

\section{Introduction}

- Dendrimers and dendritic polymers can be rationally designed and functionally tuned to control their biological interactions.

- The toxicity of amine-terminated dendrimers can be mitigated through modification of positively charged surface functional groups to charge neutral, negatively charged, or PEGylated groups.

- Cellular uptake mechanism of dendritic nanomaterials could be various and highly dependent on their size, surface group, architecture, and composition.

- Multivalent interactions enable exponentially increased ligand-receptor binding strength.

\section{Advancement of dendritic systems for drug delivery and other applications}

- Dendritic nanomaterials that incorporate multifunctionality such as therapeutic, diagnostic, and targeting capabilities have demonstrated the great potential toward clinical translation.

- Dendritic-block copolymers can combine advantages of dendrimers with those of selfassembling polymers to create hybrid nanomaterials using modular approaches.

- Alternative uses of dendrimers such as in hybrid nanoparticles and as surface-coatings for a biosensor demonstrates the versatility of dendrimer-based nanomaterials.

\section{Challenges with clinical translation of dendrimer-based nanodevices}

- Dendrimers still need to overcome issues related to their complicated, multistep synthesis, limited capacity for drug encapsulation, inability to be extensively surface modified, and in vivo efficacy.

- Dendritic nanomaterials in general need to address issues related to scalability and reproducibility.

\section{Summary}

- Dendritic nanomaterials are some of the most promising platforms for drug delivery and diagnosis.

- Choice of material composition (core, backbone, and surface functionality) is critical to design dendrimers.

- Due to differences in analysis conditions, each newly designed dendritic nanomaterial should be evaluated on an individual basis to ensure appropriate biological interactions and responses are achieved. 


\section{Bibliography}

Papers of special note have been highlighted as:

* of interest

** of considerable interest

1. Lee CC, Mackay JA, Frechet JMJ, Szoka FC: Designing dendrimers for biological applications. Nature Biotechnology 23(12), 1517-1526 (2005).

2. Esfand R, Tomalia DA: Poly(amidoamine) (PAMAM) dendrimers: from biomimicry to drug delivery and biomedical applications. Drug Discovery Today 6(8), 427-436 (2001).

3. Svenson S, Tomalia DA: Commentary - Dendrimers in biomedical applications reflections on the field. Advanced Drug Delivery Reviews 57(15), 2106-2129 (2005).

4. Gao C, Yan D: Hyperbranched polymers: from synthesis to applications. Progress in Polymer Science 29(3), 183-275 (2004).

5. Jang WD, Selim KMK, Lee CH, Kang IK: Bioinspired application of dendrimers: From bio-mimicry to biomedical applications. Progress in Polymer Science 34(1), 123 (2009).

6. Baars MWPL, Karlsson AJ, Sorokin V, De Waal BFW, Meijer EW: Supramolecular Modification of the Periphery of Dendrimers Resulting in Rigidity and Functionality. Angewandte Chemie International Edition 39(23), 4262-4265 (2000).

7. Hong S, Leroueil PR, Majoros IJ, Orr BG, Baker JR, Holl MMB: The binding avidity of a nanoparticle-based multivalent targeted drug delivery platform. Chem. Biol. 14(1), 107-115 (2007).

**Described that multivalent binding by dendrimer can increase the binding avidity of folic acid to its receptor up to 170,000 -fold.

8. Myung J, Gajjar KA, Saric J, Eddington DT, Hong S: Dendrimer-mediated multivalent binding for the enhanced capture of tumor cells. Angewandte Chemie International Edition 50(49), 11769-11772 (2011).

**Demonstrated that multivalent binding, mediated by dendrimers, could significantly enhance the capture efficiency to tumor cells under flow conditions.

9. Boas U, Heegaard PM: Dendrimers in drug research. Chemical Society Reviews 33(1), 43-63 (2004).

10. Hong S, Bielinska AU, Mecke A et al.: Interaction of poly(amidoamine) dendrimers with supported lipid bilayers and cells: Hole formation and the relation to transport. Bioconjugate Chemistry 15(4), 774-782 (2004).

11. Hong S, Rattan $\mathrm{R}$, Majoros $\mathrm{IJ}$ et al.: The role of ganglioside GM(1) in cellular internalization mechanisms of poly(amidoamine) dendrimers. Bioconjugate Chemistry 20(8), 1503-1513 (2009).

12. Patri AK, Kukowska-Latallo JF, Baker JR: Targeted drug delivery with dendrimers: Comparison of the release kinetics of covalently conjugated drug and 
non-covalent drug inclusion complex. Advanced Drug Delivery Reviews 57(15), 22032214 (2005).

13. Cheng Y, Zhao $\mathrm{L}$, Li Y, Xu T: Design of biocompatible dendrimers for cancer diagnosis and therapy: current status and future perspectives. Chemical Society Reviews 40(5), 2673-2703 (2011).

14. Heegaard PMH, Boas U, Sorensen NS: Dendrimers for Vaccine and Immunostimulatory Uses. A Review. Bioconjugate Chemistry 21(3), 405-418 (2009).

15. Tekade RK, Kumar PV, Jain NK: Dendrimers in oncology: An expanding horizon. Chemical Reviews 109(1), 49-87 (2009).

16. Khandare J, Calderon M, Dagia NM, Haag R: Multifunctional dendritic polymers in nanomedicine: opportunities and challenges. Chemical Society Reviews 41(7), 2824-2848 (2012).

17. Kaminskas LM, Porter CJH: Targeting the lymphatics using dendritic polymers (dendrimers). Advanced Drug Delivery Reviews 63(10,Äì11), 890-900 (2011).

18. Wurm F, Frey H: Linear-dendritic block copolymers: The state of the art and exciting perspectives. Progress in Polymer Science 36(1), 1-52 (2011).

19. Rosen BM, Wilson CJ, Wilson DA, Peterca M, Imam MR, Percec V: DendronMediated Self-Assembly, Disassembly, and Self-Organization of Complex Systems. Chemical Reviews 109(11), 6275-6540 (2009).

*A thorough review describing the variety of dendron-based architectures and their material properties.

20. Bae JW, Pearson RM, Patra N et al.: Dendron-mediated self-assembly of highly PEGylated block copolymers: a modular nanocarrier platform. Chemical Communications 47(37), 10302-10304 (2011).

21. Poon Z, Chen S, Engler AC et al.: Ligand-clustered "patchy" nanoparticles for modulated cellular uptake and in vivo tumor targeting. Angewandte Chemie International Edition 49(40), 7266-7270 (2010).

22. Percec V, Wilson DA, Leowanawat $P$ et al.: Self-assembly of Janus dendrimers into uniform dendrimersomes and other complex architectures. Science 328(5981), 10091014 (2010).

23. Peterca M, Percec V, Leowanawat P, Bertin A: Predicting the Size and Properties of Dendrimersomes from the Lamellar Structure of Their Amphiphilic Janus Dendrimers. Journal of the American Chemical Society, (2011).

24. Sunoqrot S, Bae JW, Pearson RM et al.: Temporal Control over Cellular Targeting through Hybridization of Folate-targeted Dendrimers and PEG-PLA Nanoparticles. Biomacromolecules 13, 1223-1230 (2012).

25. Tomalia DA, Baker H, Dewald J et al.: A New Class of Polymers - StarburstDendritic Macromolecules. Polymer Journal 17(1), 117-132 (1985).

*First extensive investigation of dendrimer structures prepared using the divergent synthetic approach. 
26. Hawker CJ, Frechet JMJ: Preparation of polymers with controlled molecular architecture. A new convergent approach to dendritic macromolecules. Journal of the American Chemical Society 112(21), 7638-7647 (1990).

*First use of convergent synthetic approach to produce dendrimers.

27. Bosman AW, Janssen HM, Meijer EW: About Dendrimers: Structure, Physical Properties, and Applications. Chemical Reviews 99(7), 1665-1688 (1999).

28. Wooley KL, Hawker CJ, Frechet JMJ: Hyperbranched macromolecules via a novel double-stage convergent growth approach. Journal of the American Chemical Society 113(11), 4252-4261 (1991).

29. Freeman AW, Fr $\sqrt{ }$ CChet JMJ: A Rapid, Orthogonal Synthesis of Poly(benzyl ester) Dendrimers via an ,ÄúActivated, Äù Monomer Approach. Organic Letters 1(4), 685688 (1999).

30. Kawaguchi T, Walker KL, Wilkins CL, Moore JS: Double Exponential Dendrimer Growth. Journal of the American Chemical Society 117(8), 2159-2165 (1995).

31. Zeng F, Zimmerman SC: Rapid Synthesis of Dendrimers by an Orthogonal Coupling Strategy. Journal of the American Chemical Society 118(22), 5326-5327 (1996).

32. Duncan R, Izzo L: Dendrimer biocompatibility and toxicity. Advanced Drug Delivery Reviews 57(15), 2215-2237 (2005).

33. Malik N, Wiwattanapatapee $\mathrm{R}$, Klopsch $\mathrm{R}$ et al.: Dendrimers: relationship between structure and biocompatibility in vitro, and preliminary studies on the biodistribution of 125I-labelled polyamidoamine dendrimers in vivo. J. Control. Release 65(1-2), 133-148 (2000).

**Demonstrated the surface-charge and generation-dependent toxicity of dendrimers.

34. Hong S, Leroueil PR, Janus EK et al.: Interaction of polycationic polymers with supported lipid bilayers and cells: Nanoscale hole formation and enhanced membrane permeability. Bioconjugate Chemistry 17(3), 728-734 (2006).

35. Jain K, Kesharwani P, Gupta U, Jain NK: Dendrimer toxicity: Let's meet the challenge. International Journal of Pharmaceutics 394(1-2), 122-142 (2010).

36. Ihre H, Padilla De Jesus OL, Frechet JM: Fast and convenient divergent synthesis of aliphatic ester dendrimers by anhydride coupling. Journal of the American Chemical Society 123(25), 5908-5917 (2001).

37. Ihre HR, Padilla De Jesus OL, Szoka FC, Jr., Frechet JM: Polyester dendritic systems for drug delivery applications: design, synthesis, and characterization. Bioconjugate Chemistry 13(3), 443-452 (2002).

38. Winnicka K, Bielawski K, Rusak M, Bielawska A: The Effect of Generation 2 and 3 Poly(amidoamine) Dendrimers on Viability of Human Breast Cancer Cells. Journal of Health Science 55(2), 169-177 (2009).

39. Padilla De Jesus OL, Ihre HR, Gagne L, Frechet JM, Szoka FC, Jr.: Polyester dendritic systems for drug delivery applications: in vitro and in vivo evaluation. Bioconjugate Chemistry 13(3), 453-461 (2002). 
40. Margerum LD, Campion BK, Koo M et al.: Gadolinium(III) DO3A macrocycles and polyethylene glycol coupled to dendrimers - Effect of molecular weight on physical and biological properties of macromolecular magnetic resonance imaging contrast agents. Journal of Alloys and Compounds 249(1-2), 185-190 (1997).

41. Owens Iii DE, Peppas NA: Opsonization, biodistribution, and pharmacokinetics of polymeric nanoparticles. International Journal of Pharmaceutics 307(1), 93-102 (2006).

42. Kojima C, Regino C, Umeda Y, Kobayashi H, Kono K: Influence of dendrimer generation and polyethylene glycol length on the biodistribution of PEGylated dendrimers. International Journal of Pharmaceutics 383(1, Ä̀̀2), 293-296 (2010).

43. Poon Z, Lee JA, Huang S, Prevost RJ, Hammond PT: Highly stable, ligandclustered "patchy" micelle nanocarriers for systemic tumor targeting. Nanomedicine 7(2), 201-209 (2011).

44. Gillies ER, Dy E, Frechet JMJ, Szoka FC: Biological Evaluation of Polyester Dendrimer: Poly(ethylene oxide) "Bow-Tie" Hybrids with Tunable Molecular Weight and Architecture. Molecular Pharmaceutics 2(2), 129-138 (2005).

45. Zhao F, Zhao Y, Liu Y, Chang X, Chen C, Zhao Y: Cellular Uptake, Intracellular Trafficking, and Cytotoxicity of Nanomaterials. Small 7(10), 1322-1337 (2011).

46. Wolinsky JB, Grinstaff MW: Therapeutic and diagnostic applications of dendrimers for cancer treatment. Advanced Drug Delivery Reviews 60(9), 1037-1055 (2008).

47. Kitchens KM, Kolhatkar IB, Swaan PW, Ghandehari H: Endocytosis inhibitors prevent poly(amidoamine) dendrimer internalization and permeability across Caco2 cells. Molecular Pharmaceutics 5(2), 364-369 (2008).

48. Albertazzi L, Serresi M, Albanese A, Beltram F: Dendrimer Internalization and Intracellular Trafficking in Living Cells. Molecular Pharmaceutics 7(3), 680-688 (2010).

49. Perumal OP, Inapagolla R, Kannan S, Kannan RM: The effect of surface functionality on cellular trafficking of dendrimers. Biomaterials 29(24-25), 34693476 (2008).

50. Rajendran L, Knolker HJ, Simons K: Subcellular targeting strategies for drug design and delivery. Nat Rev Drug Discov 9(1), 29-42 (2010).

51. Mammen M, Choi SK, Whitesides GM: Polyvalent interactions in biological systems: Implications for design and use of multivalent ligands and inhibitors. Angewandte Chemie International Edition 37(20), 2755-2794 (1998).

52. Kiessling LL, Gestwicki JE, Strong LE: Synthetic multivalent ligands in the exploration of cell-surface interactions. Current Opinion in Chemical Biology 4(6), 696-703 (2000).

53. Kiessling LL, Gestwicki JE, Strong LE: Synthetic multivalent ligands as probes of signal transduction. Angewandte Chemie International Edition 45(15), 2348-2368 (2006).

54. Vance D, Martin J, Patke S, Kane RS: The design of polyvalent scaffolds for targeted delivery. Advanced Drug Delivery Reviews 61(11), $931-939$ (2009).

55. Mecke A, Lee I, Jr JRB, Holl MMB, Orr BG: Deformability of poly(amidoamine) dendrimers. The European Physical Journal E: Soft Matter and Biological Physics 14(1), 7-16 (2004). 
56. Page D, Aravind S, Roy R: Synthesis and lectin binding properties of dendritic mannopyranoside. Chemical Communications (16), 1913-1914 (1996).

57. Ashton PR, Hounsell EF, Jayaraman N et al.: Synthesis and biological evaluation of alpha-D-mannopyranoside-containing dendrimers. Journal of Organic Chemistry 63(10), 3429-3437 (1998).

58. Kane RS: Thermodynamics of Multivalent Interactions: Influence of the Linker. Langmuir 26(11), 8636-8640 (2010).

59. Shewmake TA, Solis FJ, Gillies RJ, Caplan MR: Effects of Linker Length and Flexibility on Multivalent Targeting. Biomacromolecules 9(11), 3057-3064 (2008).

60. Kim YS, Lee JH, Ryu J, Kim DJ: Multivalent \& Multifunctional Ligands to betaAmyloid. Current Pharmaceutical Design 15(6), 637-658 (2009).

61. Rele SM, Cui WX, Wang LC et al.: Dendrimer-like PEO glycopolymers exhibit antiinflammatory properties. Journal of the American Chemical Society 127(29), 1013210133 (2005).

62. Gestwicki JE, Cairo CW, Strong LE, Oetjen KA, Kiessling LL: Influencing receptor-ligand binding mechanisms with multivalent ligand architecture. Journal of the American Chemical Society 124(50), 14922-14933 (2002).

63. Dijkgraaf I, Rijnders AY, Soede A et al.: Synthesis of DOTA-conjugated multivalent cyclic-RGD peptide dendrimers via 1,3-dipolar cycloaddition and their biological evaluation: implications for tumor targeting and tumor imaging purposes. Organic \& Biomolecular Chemistry 5(6), 935-944 (2007).

64. Menerny DQ, Kukowska-Latallo JF, Mullen DG et al.: RGD Dendron Bodies; Synthetic Avidity Agents with Defined and Potentially Interchangeable Effector Sites That Can Substitute for Antibodies. Bioconjugate Chemistry 20(10), 1853-1859 (2009).

65. Martin AL, Li B, Gillies ER: Surface Functionalization of Nanomaterials with Dendritic Groups: Toward Enhanced Binding to Biological Targets. Journal of the American Chemical Society 131(2), 734-741 (2008).

66. Vannucci L, Fiserova A, Sadalapure K et al.: Effects of N-acetyl-glucosamine-coated glycodendrimers as biological modulators in the B16F10 melanoma model in vivo. International Journal of Oncology 23(2), 285-296 (2003).

67. Fox ME, Szoka FC, Frechet JMJ: Soluble polymer carriers for the treatment of cancer: The importance of molecular architecture. Accounts of Chemical Research 42(8), 1141-1151 (2009).

68. Menjoge AR, Kannan RM, Tomalia DA: Dendrimer-based drug and imaging conjugates: design considerations for nanomedical applications. Drug Discovery Today 15(5-6), 171-185 (2010).

69. Kukowska-Latallo JF, Candido KA, Cao ZY et al.: Nanoparticle targeting of anticancer drug improves therapeutic response in animal model of human epithelial cancer. Cancer Research 65(12), 5317-5324 (2005).

**Demonstrated the marked anti-tumor activity and reduced non-specific toxicity of folic acid-targeted methotrexate-conjugated dendrimers in vivo.

70. Lim J, Chouai A, Lo S-T, Liu W, Sun X, Simanek EE: Design, Synthesis, Characterization, and Biological Evaluation of Triazine Dendrimers Bearing 
Paclitaxel Using Ester and Ester/Disulfide Linkages. Bioconjugate Chemistry 20(11), 2154-2161 (2009).

71. Zhang Y, Thomas TP, Desai A et al.: Targeted Dendrimeric Anticancer Prodrug: A Methotrexate-Folic Acid-Poly(amidoamine) Conjugate and a Novel, Rapid, "One Pot" Synthetic Approach. Bioconjugate Chemistry 21(3), 489-495 (2010).

72. Mullen DG, Desai AM, Waddell JN et al.: The implications of stochastic synthesis for the conjugation of functional groups to nanoparticles. Bioconjugate Chemistry 19(9), 1748-1752 (2008).

73. Zong H, Thomas TP, Lee K-H et al.: Bifunctional PAMAM Dendrimer Conjugates of Folic Acid and Methotrexate with Defined Ratio. Biomacromolecules Just Accepted Manuscript, DOI: 10.1021/bm201639c, (2012).

74. Okuda T, Kawakami S, Maeie T, Niidome T, Yamashita F, Hashida M: Biodistribution characteristics of amino acid dendrimers and their PEGylated derivatives after intravenous administration. J. Control. Release 114(1), 69-77 (2006).

75. Singh P, Gupta U, Asthana A, Jain NK: Folate and folate-PEG-PAMAM dendrimers: Synthesis, characterization, and targeted anticancer drug delivery potential in tumor bearing mice. Bioconjugate Chemistry 19(11), 2239-2252 (2008).

76. Wiener EC, Brechbiel MW, Brothers H et al.: Dendrimer-based metal-chelates - A new class of magnetic-resonance-imaging contrast agents Magnetic Resonance in Medicine 31(1), 1-8 (1994).

77. Malik N, Evagorou EG, Duncan R: Dendrimer-platinate: a novel approach to cancer chemotherapy. Anticancer Drugs 10(8), 767-776 (1999).

78. Baker JR, Quintana A, Piehler L, Banazak-Holl M, Tomalia D, Raczka E: The Synthesis and testing of anti-cancer therapeutic nanodevices. Biomedical Microdevices 3(1), 61-69 (2001).

79. Myc A, Douce TB, Ahuja $\mathbf{N}$ et al.: Preclinical antitumor efficacy evaluation of dendrimer-based methotrexate conjugates. Anticancer Drug 19(2), 143-149 (2008).

80. Haensler J, Szoka FC: Polyamidoamine cascade polymers mediate efficient transfection of cells in culture. Bioconjugate Chemistry 4(5), 372-379 (1993).

81. Vincent $L$, Varet J, Pille JY et al.: Efficacy of dendrimer-mediated angiostatin and TIMP-2 gene delivery on inhibition of tumor growth and angiogenesis: In vitro and in vivo studies. International Journal of Cancer 105(3), 419-429 (2003).

82. Xiao K, Li Y, Luo $\mathrm{J}$ et al.: The effect of surface charge on in vivo biodistribution of PEG-oligocholic acid based micellar nanoparticles. Biomaterials 32(13), 3435-3446 (2011).

83. D'emanuele A, Attwood D: Dendrimer-drug interactions. Advanced Drug Delivery Reviews 57(15), 2147-2162 (2005).

84. Karakoti AS, Das S, Thevuthasan S, Seal S: PEGylated Inorganic Nanoparticles. Angewandte Chemie International Edition 50(9), 1980-1994 (2011).

85. Xiao K, Li Y, Lee JS et al.: "OA02" Peptide Facilitates the Precise Targeting of Paclitaxel-Loaded Micellar Nanoparticles to Ovarian Cancer In Vivo. Cancer Research, (2012).

86. Hua C, Peng SM, Dong CM: Synthesis and characterization of linear-dendron-like poly(epsilon-caprolactone)-b-poly(ethylene oxide) copolymers via the combination of ring-opening polymerization and click chemistry. Macromolecules 41(18), 66866695 (2008). 
87. Kostiainen MA, Szilvay GR, Lehtinen J et al.: Precisely defined protein-polymer conjugates: construction of synthetic DNA binding domains on proteins by using multivalent dendrons. Acs Nano 1(2), 103-113 (2007).

88. Deng JJ, Zhou YF, Xu B, Mai KJ, Deng YB, Zhang LM: Dendronized Chitosan Derivative as a Biocompatible Gene Delivery Carrier. Biomacromolecules 12(3), 642-649 (2011).

89. Barnard A, Posocco P, Pricl S et al.: Degradable Self-Assembling Dendrons for Gene Delivery: Experimental and Theoretical Insights into the Barriers to Cellular Uptake. Journal of the American Chemical Society 133(50), 20288-20300 (2011).

90. Wood KC, Little SR, Langer R, Hammond PT: A family of hierarchically selfassembling linear-dendritic hybrid polymers for highly efficient targeted gene delivery. Angewandte Chemie International Edition 44(41), 6704-6708 (2005).

91. Yu HJ, Nie Y, Dohmen C, Li YQ, Wagner E: Epidermal Growth Factor-PEG Functionalized PAMAM-Pentaethylenehexamine Dendron for Targeted Gene Delivery Produced by Click Chemistry. Biomacromolecules 12(6), 2039-2047 (2011).

92. Wood KC, Azarin SM, Arap W, Pasqualini R, Langer R, Hammond PT: Tumortargeted gene delivery using molecularly engineered hybrid polymers functionalized with a tumor-homing peptide. Bioconjugate Chemistry 19(2), 403-405 (2008).

93. Pantel K, Brakenhoff RH: Dissecting the metastatic cascade. Nature Reviews Cancer 4(6), 448-456 (2004).

94. Ring A, Smith IE, Dowsett M: Circulating tumour cells in breast cancer. Lancet Oncology 5(2), 79-88 (2004).

95. Nagrath S, Sequist LV, Maheswaran S et al.: Isolation of rare circulating tumour cells in cancer patients by microchip technology. Nature 450(7173), 1235-U1210 (2007).

96. Stott SL, Hsu CH, Tsukrov DI et al.: Isolation of circulating tumor cells using a microvortex-generating herringbone-chip. Proceedings of the National Academy of Sciences of the United States of America 107(43), 18392-18397 (2010).

97. Lee RT, Lee YC: Affinity enhancement by multivalent lectin-carbohydrate interaction. Glycoconjugate Journal 17(7-9), 543-551 (2000).

98. Mourez M, Kane RS, Mogridge $J$ et al.: Designing a polyvalent inhibitor of anthrax toxin. Nature Biotechnology 19(10), 958-961 (2001).

99. He W, Wang HF, Hartmann LC, Cheng JX, Low PS: In vivo quantitation of rare circulating tumor cells by multiphoton intravital flow cytometry. Proceedings of the National Academy of Sciences of the United States of America 104(28), 11760-11765 (2007).

100. Dimitroff CJ, Lechpammer M, Long-Woodward D, Kutok JL: Rolling of human bone-metastatic prostate tumor cells on human bone marrow endothelium under shear flow is mediated by E-selectin. Cancer Research 64(15), 5261-5269 (2004).

101. Mcneeley KM, Annapragada A, Bellamkonda RV: Decreased circulation time offsets increased efficacy of PEGylated nanocarriers targeting folate receptors of glioma. Nanotechnology 18(38), (2007).

102. Mcneeley KM, Karathanasis E, Annapragada AV, Bellamkonda RV: Masking and triggered unmasking of targeting ligands on nanocarriers to improve drug delivery to brain tumors. Biomaterials 30(23-24), 3986-3995 (2009). 
103. Gabizon A, Horowitz AT, Goren D, Tzemach D, Shmeeda H, Zalipsky S: In vivo fate of folate-targeted polyethylene-glycol liposomes in tumor-bearing mice. Clinical Cancer Research 9(17), 6551-6559 (2003).

104. Danhier F, Feron O, Preat V: To exploit the tumor microenvironment: Passive and active tumor targeting of nanocarriers for anti-cancer drug delivery. $J$. Control. Release 148(2), 135-146 (2010).

105. Patri AK, Majoros IJ, Baker Jr JR: Dendritic polymer macromolecular carriers for drug delivery. Current Opinion in Chemical Biology 6(4), 466-471 (2002).

106. Kukowska-Latallo JF, Candido KA, Cao $\mathrm{Z}$ et al.: Nanoparticle Targeting of Anticancer Drug Improves Therapeutic Response in Animal Model of Human Epithelial Cancer. Cancer Research 65(12), 5317-5324 (2005).

107. Zhu S, Hong M, Zhang L, Tang G, Jiang Y, Pei Y: PEGylated PAMAM DendrimerDoxorubicin Conjugates: In Vitro Evaluation and In Vivo Tumor Accumulation. Pharmaceutical Research 27(1), 161-174 (2010).

108. Yang WL, Barth RF, Wu G et al.: Convection enhanced delivery of boronated EGF as a molecular targeting agent for neutron capture therapy of brain tumors. $J$ Neuro-Oncol 95(3), 355-365 (2009).

109. Choe YH, Conover CD, Wu DC et al:: Anticancer drug delivery systems: multiloaded N-4-acyl poly(ethylene glycol) prodrugs of ara-C. II. Efficacy in ascites and solid tumors. J. Control. Release 79(1-3), 55-70 (2002).

110. Pasut G, Scaramuzza S, Schiavon O, Mendichi R, Veronese FM: PEG-epirubicin conjugates with high drug loading. Journal of Bioactive and Compatible Polymers 20(3), 213-230 (2005).

111. Fox ME, Guillaudeu S, Frechet JM, Jerger K, Macaraeg N, Szoka FC: Synthesis and in vivo antitumor efficacy of PEGylated poly(l-lysine) dendrimer-camptothecin conjugates. Mol Pharm 6(5), 1562-1572 (2009).

112. Van Der Poll DG, Kieler-Ferguson HM, Floyd WC et al.: Design, Synthesis, and Biological Evaluation of a Robust, Biodegradable Dendrimer. Bioconjugate Chemistry 21(4), 764-773 (2010).

113. Dhanikula RS, Argaw A, Bouchard JF, Hildgen P: Methotrexate loaded polyethercopolyester dendrimers for the treatment of gliomas: Enhanced efficacy and intratumoral transport capability. Molecular Pharmaceutics 5(1), 105-116 (2008).

114. Taratula O, Garbuzenko OB, Kirkpatrick $P$ et al.: Surface-engineered targeted PPI dendrimer for efficient intracellular and intratumoral siRNA delivery. Journal of Controlled Release 140(3), 284-293 (2009).

115. Defoort JP, Nardelli B, Huang WL, Ho DD, Tam JP: Macromolecular assemblage in the design of a synthetic AIDS vaccine. Proceedings of the National Academy of Sciences of the United States of America 89(9), 3879-3883 (1992).

116. Bourne N, Stanberry LR, Kern ER, Holan G, Matthews B, Bernstein DI: Dendrimers, a new class of candidate topical microbicides with activity against herpes simplex virus infection. Antimicrobial Agents and Chemotherapy 44(9), 24712474 (2000).

117. Jiang YH, Emau P, Cairns JS et al.: SPL7013 gel as a topical microbicide for prevention of vaginal transmission of SHIV89.6P in macaques. Aids Research and Human Retroviruses 21(3), 207-213 (2005). 
118. Bernstein DI, Bourne N, Ayisi NK et al.: Evaluation of formulated dendrimer SPL7013 as a microbicide. Antiviral Research 57(3), A66-A66 (2003).

119. Al-Jamal KT, Al-Jamal WT, Akerman S et al.: Systemic antiangiogenic activity of cationic poly-L-lysine dendrimer delays tumor growth. Proceedings of the National Academy of Sciences of the United States of America 107(9), 3966-3971 (2010).

120. Olson ES, Jiang T, Aguilera TA et al.: Activatable cell penetrating peptides linked to nanoparticles as dual probes for in vivo fluorescence and MR imaging of proteases. P Natl Acad Sci USA 107(9), 4311-4316 (2010).

121. Cheng $Z$, Thorek DL, Tsourkas A: Gadolinium-conjugated dendrimer nanoclusters as a tumor-targeted $\mathrm{T} 1$ magnetic resonance imaging contrast agent. Angew Chem Int Edit 49(2), 346-350 (2010).

122. Kobayashi H, Koyama Y, Barrett $\mathbf{T}$ et al.: Multimodal nanoprobes for radionuclide and five-color near-infrared optical lymphatic imaging. Acs Nano 1(4), 258-264 (2007).

123. Kaneshiro TL, Jeong EK, Morrell G, Parker DL, Lu ZR: Synthesis and Evaluation of Globular Gd-DOTA-Monoamide Conjugates with Precisely Controlled Nanosizes for Magnetic Resonance Angiography. Biomacromolecules 9(10), 2742-2748 (2008).

124. Herborn CU, Barkhausen J, Paetsch I et al.: Coronary arteries: Contrast-enhanced MR imaging with SH L 643A - Experience in 12 volunteers. Radiology 229(1), 217223 (2003).

125. Chisholm EJ, Vassaux G, Martin-Duque $P$ et al.: Cancer-Specific Transgene Expression Mediated by Systemic Injection of Nanoparticles. Cancer Research 69(6), 2655-2662 (2009).

126. Parrott MC, Benhabbour SR, Saab C et al.: Synthesis, Radiolabeling, and Bioimaging of High-Generation Polyester Dendrimers. Journal of the American Chemical Society 131(8), 2906-2916 (2009).

127. Almutairi A, Rossin $\mathrm{R}$, Shokeen $\mathrm{M}$ et al.: Biodegradable dendritic positron-emitting nanoprobes for the noninvasive imaging of angiogenesis. P Natl Acad Sci USA 106(3), 685-690 (2009).

128. Tian L, Hammond PT: Comb-dendritic block copolymers as tree-shaped macromolecular amphiphiles for nanoparticle self-assembly. Chemistry of Materials 18(17), 3976-3984 (2006).

129. Gillies ER, Frechet JM: Designing macromolecules for therapeutic applications: polyester dendrimer-poly(ethylene oxide) "bow-tie" hybrids with tunable molecular weight and architecture. Journal of the American Chemical Society 124(47), 1413714146 (2002).

130. Demattei CR, Huang B, Tomalia DA: Designed Dendrimer Syntheses by SelfAssembly of Single-Site, ssDNA Functionalized Dendrons. Nano Letters 4(5), 771777 (2004). 


\section{Copy of Reference Annotations (6-8 refs) (1-2 line synopsis)}

Key (per guidelines)

* of interest

$* *$ of considerable interest

1) Hong, S., Leroueil, P.R., Majoros, I.J., Orr, B.G., Baker, J.R., Holl, M.M.B. (2007). The binding avidity of a nanoparticle-based multivalent targeted drug delivery platform. Chem. Biol., 14, 107-115.

**Described that multivalent binding by dendrimer can increase the binding avidity of folic acid to its receptor up to 170,000 -fold.

2) Malik, N., Wiwattanapatapee, R., Klopsch, R., Lorenz, K., Frey, H., Weener, J.W., Meijer, E.W., Paulus, W., Duncan, R. (2000). Dendrimers: relationship between structure and biocompatibility in vitro, and preliminary studies on the biodistribution of 125I-labelled polyamidoamine dendrimers in vivo. Journal of Controlled Release, 65, 133-48.

**Demonstrated the surface-charge and generation-dependent toxicity of dendrimers.

3) Tomalia, D.A., Baker, H., Dewald, J., Hall, M., Kallos, G., Martin, S., Roeck, J., Ryder, J., Smith, P. (1985). A New Class of Polymers - Starburst-Dendritic Macromolecules. Polymer Journal, 17, 117-132.

*First extensive investigation of dendrimer structures prepared using the divergent synthetic approach.

4) Hawker, C.J., Frechet, J.M.J. (1990). Preparation of polymers with controlled molecular architecture. A new convergent approach to dendritic macromolecules. Journal of the American Chemical Society, 112, 7638-7647.

*First use of convergent synthetic approach to produce dendrimers.

5) Rosen, B.M., Wilson, C.J., Wilson, D.A., Peterca, M., Imam, M.R., Percec, V. (2009). Dendron-Mediated Self-Assembly, Disassembly, and Self-Organization of Complex Systems. Chemical Reviews, 109, 6275-6540.

*A thorough review describing the variety of dendron-based architectures and their material properties.

6) Myung, J., Gajjar, K.A., Saric, J., Eddington, D.T., Hong, S. (2011). Dendrimer-mediated multivalent binding for the enhanced capture of tumor cells. Angew. Chem. Int. Ed., 50, 1176911772 .

**Demonstrated that multivalent binding, mediated by dendrimers, could significantly enhance the capture efficiency to tumor cells under flow conditions. 
7) Kukowska-Latallo, J.F., Candido, K.A., Cao, Z., Nigavekar, S.S., Majoros, I.J., Thomas, T.P., Balogh, L.P., Khan, M.K., Baker, J.R. (2005). Nanoparticle Targeting of Anticancer Drug Improves Therapeutic Response in Animal Model of Human Epithelial Cancer. Cancer Research, $65,5317-5324$.

**Demonstrated the marked anti-tumor activity and reduced non-specific toxicity of folic acid-targeted methotrexate-conjugated dendrimers in vivo. 